\title{
Fístula extraoral asociada a sialolitiasis en el conducto de Wharton. Reporte de un caso
}

\section{Extra-Oral Fistula Associated with Sialolithiasis in Whartons duct. Case Report}

\author{
Danny Obando Solano DDS¹; José Manuel Fernández Chaves DDS² \\ 1. Odontólogo General, Universidad de Costa Rica. Pasantía en Cirugía Oral y Oseointegración. \\ Facultad de Odontología, Universidad de Costa Rica, Costa Rica. \\ 2. Odontólogo Especialista en Patología y Cirugía Oral. Odontología Legal y Forense. Pontificia Universidad \\ Javeriana. Profesor Instructor Pasantía en Cirugía Oral y Oseointegración.
} Facultad de Odontología, Universidad de Costa Rica, Costa Rica.

Autor para correspondencia: Dr. Danny Obando Solano - dannyobandodds@gmail.com

Recibido: 13-V-2014

Aceptado: 01-XI-2014

Publicado Online First: 15-XI-2015

DOI: http://dx.doi.org/10.15517/ijds.v0i0.22143

\section{RESUMEN}

La sialolitiasis es una de las patologías que afectan con mayor frecuencia las glándulas salivales, y es en la glándula submandibular donde se presenta más comúnmente debido a sus características anatómicas. Existen muchas teorías que explican su etiología, pero aún no está completamente determinada. La sintomatología más común es la presencia de dolor, especialmente a la hora de comer debido a la estimulación salival producida por los alimentos. También se presenta inflamación del piso de la boca e infección, asociadas a procesos de sialadenitis crónica, la formación de fístulas cutáneas es muy poco frecuente. Se reporta el caso de un paciente masculino de 31 años quien presenta una fístula extraoral en lado izquierdo su cuello, a nivel de subzona lb (submandibular) con una sialadenitis crónica asociada a sialolitiasis en el conducto de Wharton izquierdo con una evolución de cuatro años. Se realiza la descripción del manejo quirúrgico y se hace una revisión de la literatura.

\section{PALABRAS CLAVE}

Fístula extraoral, Sialolitiasis, Conducto de Wharton, Glándula submandibular. 


\section{ABSTRACT}

Sialolithiasis is one of the frecuent pathologies that affect the salivary glands, and the submandibular gland is the most commonly affected due to it's anatomy. There are many theories that try to explain the etiology, but is not yet fully determined. The most common symptom is the presence of pain, especially when eating because of the salivary stimulation produced by food. Other symptoms seen are the inflammation of the floor of mouth and infection associated with chronic sialadenitis processes; the formation of cutaneous fistulas is rare. This case report presents a 31-year-old male patient with an extra-oral fistula located on the left side of his neck subzone lb (submandibular); with chronic sialadenitis associated with sialolithiasis in the left Wharton's duct with a four-year progress. The surgical management is described and a review of the literature is performed.

\section{KEYWORDS}

Extra, Oral fistulae, Sialolitiasis, Wharton's duct, Submandibular gland.

\section{INTRODUCCIÓN}

Las glándulas submandibular, parótida y sublingual conforman las glándulas salivales mayores que son pares. La glándula submandibular se ubica en la parte posterior del piso de la boca, su secreción de tipo mixta (mucoserosa) es liberada en la cavidad bucal a través del conducto de Wharton. Dicho conducto desemboca en la boca a través de un pequeño orificio llamado carúncula sublingual, que se ubica bilateralmente al lado del frenillo lingual.

Izurieta et al (1) describen la formación de cálculos en vías excretoras (urinaria, biliar, salival, lacrimal o pancreática) como "litiasis".

Jiménez \& Reyes (2), Escudier \& Mcgurk (3) y Purohit et al (4) indican que la sialolitiasis es una de las patologías más comunes de las glándulas salivales, afectando al $1.2 \%$ de la población, y representa el $11 \%$ de todas las patologías asociadas a las glándulas salivales mayores de acuerdo con Prado et al (5).

Okada et al. (6) al igual que Rebolledo et al (7) afirman que está afección no neoplásica, es definida como una obstrucción mecánica de la glándula salival o de su conducto excretor por la formación de cálculos, concreciones calcáreas 0 sialolitos en el parénquima. Existen microcálculos asintomáticos en las glándulas salivales, que se generan constantemente, los cuales tienden a eliminarse espontáneamente, por sí solos de acuerdo con labal et al (8). Siddiqui (9) asevera que los sialolitos ocasionan obstrucción de la saliva, especialmente cuando es estimulada por los alimentos, asociándose a una inflamación recurrente durante las comidas. Esto genera dolor, debido a la estimulación del flujo salival y exacerba los síntomas preexistentes señalan Chandak \& Degwekar (10) aunque en un $17 \%$ de los casos la afección es asintomática según Oteri et al. (11).

Bernardon et al (12) afirman que al haber persistencia de la condición podría generarse una sialolitiasis crónica, con sintomatología dolorosa, inflamación y drenaje de exudado purulento, caracterizado por un cuadro de sialoadenitis. Teymoortash et al (13) mencionan que la sialolitiasis es la causa mas común de sialadenitis obstructiva.

\section{REPORTE DE CASO}

Se presenta a la clínica de Exodoncia y Cirugía de la Universidad de Costa Rica un paciente masculino de 31 años de edad, sin antecedentes patológicos personales, con inflamación en 
cuello del lado izquierdo, y con dolor agudo de aproximadamente una semana de evolución, quien además presenta una fístula extraoral. El paciente menciona que "desde hace más o menos cuatro años tengo inflamación en el suelo de la boca cuando me toco sale pus bajo la lengua, y en el orificio en el cuello me sale algo que parece saliva, y me duele al comer y tragar".

Debido a que el paciente labora como chef, el calor de la cocina y la estimulación de la saliva debido a las comidas, hace que la inflamación y el dolor asociados sean constantes.

Una semana antes de su primer visita, el paciente visitó a su odontólogo general, quien le tomó una radiografía periapical, en la cual se evidenciaba la presencia de una estructura radiopaca a nivel de molares inferiores izquierdas por ello es referido a la Pasantía de Cirugía Oral y Oseointegración para su valoración.

Al examen clínico se observa inflamación del lado izquierdo del piso de boca, y por la carúncula (el orificio de salida del conducto de Wharton) del mismo lado no hay drenaje de saliva. A la hora de palpar se detecta una zona indurada a nivel de la segunda y la tercer molares inferiores izquierdas con salida de material purulento por el conducto, $y$ presencia de dolor.

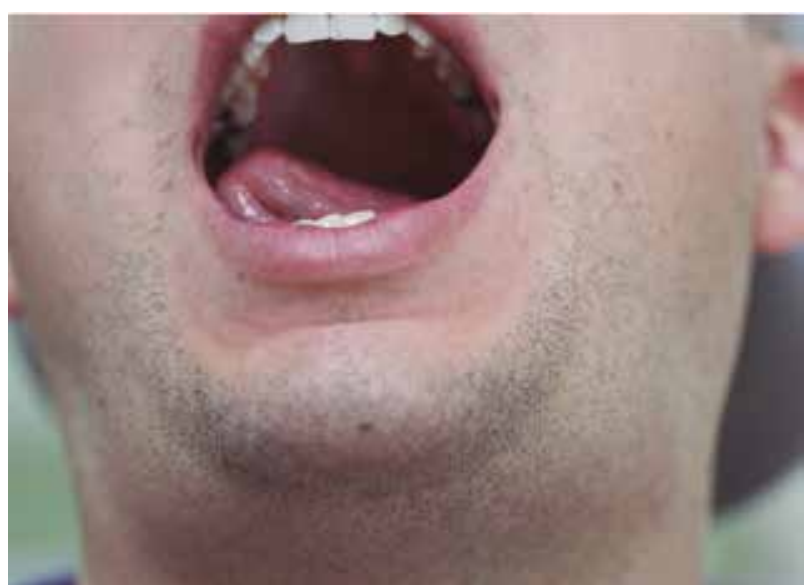

Figura 1. Fotografía extraoral.
Se solicita un ultrasonido, en cuyo reporte se describe una glándula submandibular izquierda aumentada de tamaño, con dilatación de su conducto principal, pero sin lograr identificar la causa de la obstrucción, siendo una Sialoadenitis el diagnóstico emitido. En la radiografía panorámica no se logra observar ninguna patología.

Se realiza una radiografía oclusal, en la cual se observa la presencia de una estructura radiopaca circular en el lado izquierdo del piso de la boca, de aproximadamente $6 \mathrm{~mm}$ de diámetro. Con base en las imágenes radiológicas, y las características clínicas se diagnostica una Sialoadenitis crónica asociada a Sialolitiasis en la glándula submandibular izquierda.

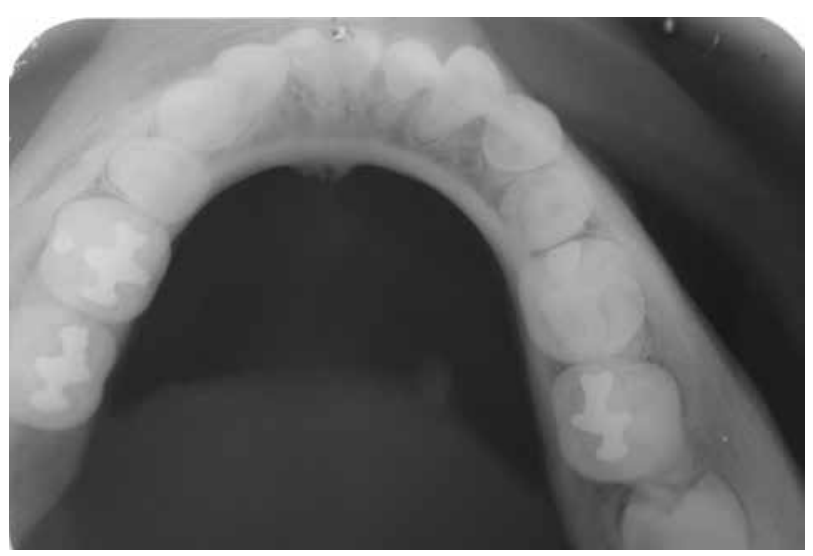

Figura 2. Radiografía oclusal.

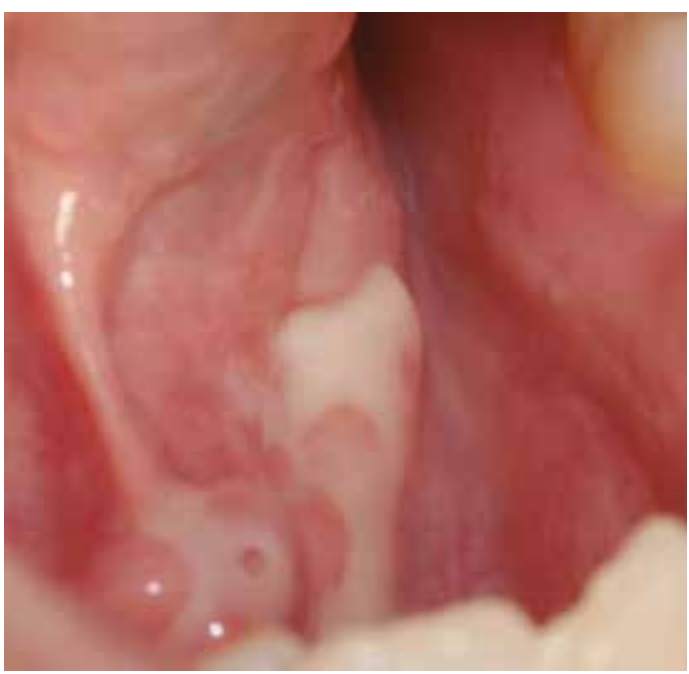

Figura 3. Secreción purulenta. 
Debido al proceso infeccioso, se prescribe terapia antibiótica con Levofloxacina 500mg dos veces al día por 7 días., para disminuir la infección y ver la evolución. Se decide realizar drenaje del conducto en la semana siguiente y posteriormente realizar una cirugía para extirpar el sialolito.

Una vez finalizado el esquema antibiótico de 7 días el paciente se presenta a la clínica refiriendo una leve mejoría, pero aún persiste el dolor y la salida de líquido purulento por el orificio del conducto. Se realiza el drenaje del material purulento, bajo anestesia local, a través del conducto de Wharton, introduciendo un catéter pediátrico por medio de su orificio de salida. También se observa drenaje de material calcificado. De esta manera se disminuye la inflamación y el dolor ocasionados por la presencia de la infección. Se continúa con terapia antibiótica, y se programa la cirugía para la siguiente semana.

El día de la cirugía, se realiza incisión en la parte posterior del conducto, en la zona donde se localiza la inflamación. Se observa salida de pus. Debido al tiempo de evolución, las paredes del conducto en la zona del abordaje estaban atrofiadas, Io que dificultó la localización del sialolito. Una vez que se detectó, se procedió con su extracción. Se realizó lavado profuso de la herida con suero fisiológico, y se dejó un drenaje de Penrose suturándolo en las paredes del conducto para evitar estenosis del mismo y dejando además el conducto abierto para que se realice su cicatrización permitiendo el drenaje del material purulento y flujo salival.

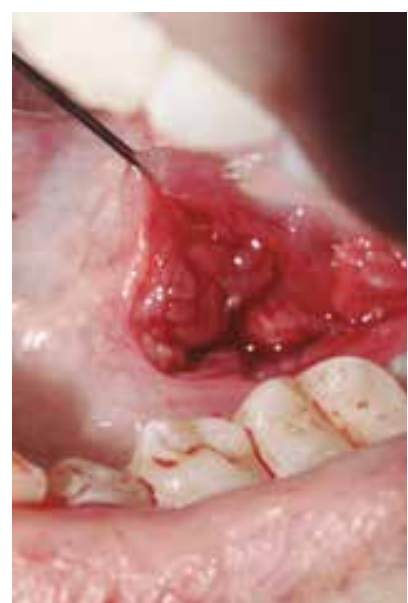

Figura 4. Incisión.

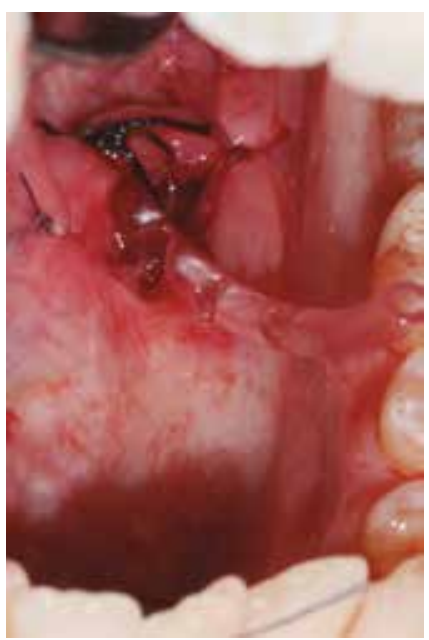

Figura 6. Colocación de dren.

La estructura que se extrajo era de consistencia suave, y de color amarillento. De forma alargada con un tamaño de $6 \mathrm{~mm} \times 3 \mathrm{~mm}$. Se envía a estudio histopatológico, y el resultado obtenido es "material hialino amorfo con exudado inflamatorio agudo, compatible con sialolito". Se confirma el diagnóstico de Sialolitiasis en el conducto de Wharton de la Glándula Submandibular. 
A la semana siguiente, el paciente refiere mejoría pero aun manifiesta dolor leve y drenaje de pus a través de la herida, y ya no se observa inflamación. Tres semanas después de la cirugía, ya no hay salida de material purulento, el dolor ha disminuido considerablemente y el conducto se encuentra permeable. Se realiza control postoperatorio cuatro semanas después, y se observa cicatrización satisfactoria de la herida, sin inflamación, ausencia de pus, no hay dolor a la palpación y el flujo salival es normal.

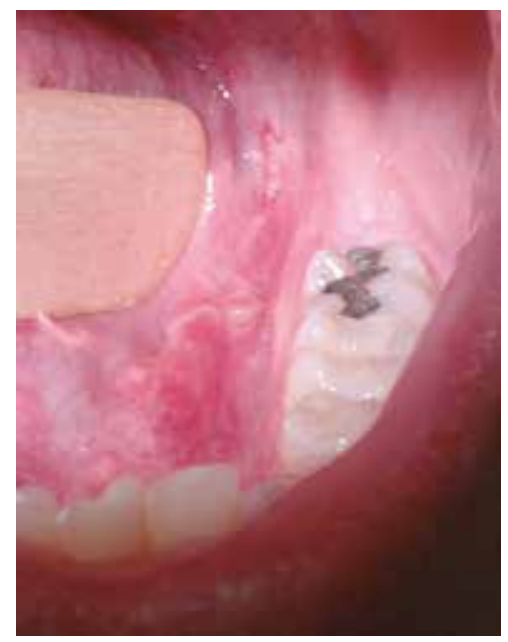

Figura 7. Control postoperatorio.

\section{DISCUSIÓN}

La deposición de sales de calcio ocurre usualmente en hueso, pero cuando ocurre de manera desorganizada en tejido blando se conoce como una calcificación heterotópica, la cual es idiopática cuando los niveles de calcio y fósforo se encuentran dentro de los valores normales. Los sialolitos pertenecen a esta categoría de acuerdo con Prabhat (14).

\section{INCIDENCIA}

Saluja et al. (15) indican que la sialolitiasis tiene una incidencia de $1,2 \%$ en la población adulta, afectando en una relación 2:1 de hombres con respecto a las mujeres, y se presenta con más frecuencia entre la tercera y sexta década de vida según Tomomichi et al (16), siendo raro en niños (3\% de los casos) indican Oteri et al. (11).

Crippa \& Calcagnile (17) estiman que el $90 \%$ de los casos se presentan en las glándulas submandibulares, un $6 \%$ en la glándula parótida, y únicamente el $2 \%$ afecta las glándulas sublinguales y salivales menores. Cuando se presenta en estas últimas afecta principalmente a las localizadas en la mucosa yugal y el labio superior según Saluja et al. (15).

Purohit et al. (4) refieren que las razones probables por las que la sialolitiasis afecta con más frecuencia a la glándula submandibular, son las siguientes: su localización anatómica, su contorno largo y tortuoso y por ser el orificio de salida más estrecho que el conducto. Además, Oteri et al. (11) indican que el flujo salival viaja en un sentido antigravitacional. El lugar más común donde se presentan los sialolitos, es donde el conducto se dobla alrededor del borde distal del músculo milohioideo, y distal al orificio del conducto según Shetty \& Sharma (18).

\section{ETIOPATOLOGÍA}

Kopec et al. (19) mencionan que la etiología y patogénesis exactas de los cálculos salivales son desconocidas, pero se han descrito diversas teorías que podrían explicar la causa de esta patología. Se sugiere que bajo circunstancias normales, se pueden formar microsialolitos, pero estos son lavados espontáneamente y removidos naturalmente por la salida del conducto de la glándula.

Los sialolitos se forman por la mineralización de cuerpos extraños, células epiteliales descamadas y microorganismos que se disponen en un nido, debido a la presencia de soluciones de continuidad como traumatismos que dificulten el flujo salival 0 a hipersaturación de la saliva con calcio y fósforo. También se mencionan infecciones bacterianas, virales o fúngicas e irritación por 
cuerpos extraños como posibles razones que ocasionan que el fosfato tricálcico amorfo se cristalice, y se transforme en hidroxiapatita provocando la formación del cálculo de acuerdo con Rebolledo et al (7).

Oteri et al. (11) establece que las toxinas producidas por bacterias que ocasionan infecciones, pueden establecer un medio con pH menor a 5,5 (medio ácido) que provoca daño tisular; cuando se restablece el pH a 7,2 (promedio normal) debido al proceso de sanado, ocurre la cristalización de iones salivales, principalmente los fosfatos cálcicos.

Entre otras posibles causas se incluyen las siguientes. La utilización de medicamentos que inhiben 0 disminuyen la salivación (antihistamínicos, diuréticos o antipsicóticos) 0 en pacientes con hiperparatiroidismo secundario a falla renal crónica afirman Peterson \& Murphy (20). Purohit et al (4) menciona que la gota es una enfermedad sistémica que predispone la formación de sialolitos predominantemente compuestas por ácido úrico Por otra parte, en un $10 \%$ de los pacientes con nefrolitiasis se reporta la aparición de sialolitiasis de acuerdo con Jiménez et al. (2).

Prabhat (14) menciona una teoría que propone que sustancias 0 bacterias en la cavidad oral podrían migrar al conducto salival y formar el nido para que se produzca la calcificación (teoría retrograda). Iqbal et al. (8) afirma que la saliva alcalina, rica en mucina contribuye a la formación de cálculos salivares. Marchal et al (21) refieren que el estancamiento de la saliva, el aumento de la alcalinidad salival, infecciones 0 inflamaciones del conducto 0 de la glándula, el trauma y el fumado de tabaco podrían predisponer a la formación del cálculo.

Según lo refieren Rebolledo et al. (7) e Iqbal et al. (8), la sialolitiasis es más común del lado izquierdo, y rara vez es bilateral. La glándula submandibular forma sialolitos alargados, los cuales se presentan más en el conducto (intraductales) que en el parénquima glandular (intraglandulares) otros autores como Saluja et al (15), Marwaha \& Nanda (22) mencionan que un tercio de los casos se presentan en el tercio distal del conducto. Mayormente se presentan como sialolitos individuales (70-80\%), pero también pueden aparecer dos en el 20\% de los casos, y en $5 \%$ hay tres o más sialolitos asociados afirman Saluja et al. (15).

Los sialolitos crecen en promedio $1-1.5 \mathrm{~mm}$ por año dice Saluja et al (15). Pueden estar libres 0 adheridos a la pared del conducto según Baptista et al (23). Presentan variedad de formas, desde redondeadas hasta ovaladas mencionan Marwaha \& Nanda (22) y pueden tener diversos tamaños, desde menos de un milímetro hasta algunos centímetros en diámetro o más grandes de acuerdo con Oteri et al (11). En las radiografías podrían observarse con formas semejantes a los dientes.

Cuando el cálculo está localizado en un conducto capaz de dilatarse y permitir secreción normal de saliva alrededor del cálculo, el sialolito puede aumentar de tamaño convirtiéndose en un cálculo gigante y mantenerse asintomático por mucho tiempo según señala Oteri et al. (11). Se han reportado casos de sialolitos de hasta 6, 7 y $9 \mathrm{~cm}$ asociados a glándula submandibular, aunque usualmente los cálculos mayores a $3 \mathrm{~cm}$, conocidos como sialolitos gigantes, son raros afirma Shingal et al. (24).

Prabhat (14) describe que los cálculos submandibulares consisten en un $82 \%$ en material inorgánico y en un $18 \%$ un material orgánico, en contraste los cálculos parotídeos se componen en un $49 \%$ de material inorgánico y en un $51 \%$ orgánico. Marchal \& Dulguerov (25) y Williams (26) mencionan que el material inorgánico se compone de fosfatos de calcio, carbonatos en forma de hidroxiapatita, magnesio, potasio y amonio. E material orgánico consiste de carbohidratos y 
aminoácidos, glicoproteínas, mucopolisacáridos y restos celulares.

Dalkiz et al. (27) indican que la saliva retenida ejerce presión retrograda en la glándula salival, y en el sistema ductal. La obstrucción que se genera podría ser parcial o total, generando una inflamación del piso de la boca según Crippa, (16). Durante las horas de las comidas, se produce dolor e inflamación, debido a esta obstrucción menciona Prabhat (14). La obstrucción no tratada, a largo plazo podría llevar a la atrofia glandular, resultando en falta de actividad secretoria y finalmente fibrosis según Siddiqui (9).

El paciente podría presentar un cólico salival, el cual consiste en un dolor agudo, lacerante y de corta duración que desaparece 15-20 minutos después de su manifestación inicial describen Marwaha \& Nanda (22). En este caso hay inflamación dolorosa producida por la retención total de saliva y el espasmo del conducto indica Izurieta et al. (1), además de una sensación de presión en el piso de la boca según Oteri et al. (11).

\section{DIAGNÓSTICO}

Siddiqui (9) puntualiza que para poder detectar y diagnosticar los sialolitos lo primero que se debe realizar es una buena historia clínica y una examinación cuidadosa. Lemos \& Camargo (28) sugieren la palpación bimanual del piso de la boca en sentido posteroanterior, la cual revelará un nódulo indurado en el piso de la boca, correspondiendo a un cálculo palpable.

Además, Prabhat (14) considera que la obtención de imágenes radiológicas es muy útil para su diagnóstico, como por ejemplo la radiografía oclusal, la radiografía panorámica, o la radiografía lateral. Prado et al. (5) mencionan que la radiografía oclusal es el método más sencillo y económico para diagnosticar un cálculo salival, especialmente cuando es palpable a nivel del segundo molar mandibular.

Típicamente aparecen en un examen radiográfico como masas radiopacas resalta Purohit (4), pero un $20 \%$ de los sialolitos asociados a la glándula submandibular aparecen radiolúcidos. Otros medios como el CT Scan (Computed Tomograhy Scan), ultrasonido (ecografía), sialografía, resonancia magnética nuclear, y sialendoscopía pueden ser útiles como estudios imagenológicos adicionales cuando hay señales de sialadenitis relacionada con sialolitos radiolúcidos afirman Marchal \& Dulguerov (25).

Se debe tomar en cuenta que un ultrasonido no siempre conseguirá detectar los sialolitos si no logran generar sombras acústicas. Pero Siddiqui (9) y Oteri et al. (11), alertan que no debe realizarse una sialografía en casos de infección, en pacientes alérgicos al material de contraste 0 en casos en que el sialolito se encuentre en la porción distal del conducto pues podría desplazar el sialolito a la porción proximal (hacia la glándula). La tomografía es el método diagnóstico más efectivo, pero su inconveniente es su alto costo, en comparación con otros medios diagnósticos mencionan Shetty \& Sharma (18) además que los sialolitos raramente se acompañan de fístulas intraorales o cutáneas.

Sreetharam \& Philip (29) mencionan que el diagnóstico diferencial de sialolitiasis incluye al nódulo linfático calcificado, a un diente retenido, a un cuerpo extraño, a un flebolito (cálculo en venas varicosas) y a la miositis osificante.

\section{TRATAMIENTO}

Existen diferentes opciones para manejar una sialolitiasis. De acuerdo a Prado et al. (5), el tratamiento depende del compromiso de la funcionalidad de la glándula según su cronicidad de la inflamación, localización y tamaño del 
sialolito. Cuando no son tratados a tiempo, podrían exfoliarse solos en el piso de la boca, ulcerar el conducto y perforar el piso de la boca, o asociarse con fístulas cutáneas por infección supurativa menciona Shingal et al (24).

Como 10 indican Marchal \& Dalguerov (25), el objetivo del tratamiento es restaurar la secreción salival normal. El manejo conservador, especialmente en sialolitos pequeños, es beneficioso. Es de excelente ayuda que el paciente esté bien hidratado y utilice sialogogos que aumenten el flujo salival, y que el clínico aplique calor húmedo en la zona, además de realizar un masaje ("ordeñar") en la glándula y el conducto para intentar extraer el sialolito por el orificio del conducto señala Chandak et al. (10). El tratamiento clásico es el uso de antibióticos antiestafilococos preferiblemente, debido a su asociación con sialadenitis, y también agentes antiinflamatorios y analgésicos resalta Prabhat (14).

Bernardon et al (12) y Marwaha \& Nanda (22) indican que el uso de calor externo, calor interno y enjuagues con jugo de limón o chupar confites o caramelos de naranja o limón, pueden ayudar al desplazamiento y expulsión espontánea de los sialolitos, por la estimulación del flujo salival. El cateterismo por medio de sondas lacrimales introducidas dentro del orificio facilitan la dilatación del conducto y permite su remoción.

En sialolitos más grandes se requiere la exposición quirúrgica del mismo (sialolitectomía), la cual se puede posponer en el estado agudo, siendo esencial la terapia antibiótica hasta que el dolor y la inflamación disminuyan. Pero también podría realizarse inmediatamente, aunque sea un proceso agudo, pues de esta manera se facilitará el alivio inmediato de la presión por medio del drenaje del exudado purulento y liberando la saliva retenida describe Dalkiz et al. (27). La técnica consiste en realizar una incisión en la mucosa directamente sobre el sialolito. Se podría previamente sujetar el conducto con una sutura, posterior a la ubicación del sialolito, para evitar su desplazamiento en sentido posterior cuando se realice la incisión. Usualmente no es necesario suturar la herida, permitiendo que el conducto sane espontáneamente mencionan Shetty \& Sharma (18).

Marchal \& Dulguerov (25) indican que otra técnica es combinar la sialolitectomía con una sialodocoplastía o marsupialización, realizando una incisión para extraer el sialolito, y suturar los bordes del conducto en los bordes de la herida lo cual permitiría el drenaje del conducto formando un nuevo orificio que funcione como baipás (en inglés bypass) del flujo salival. Se ha reportado que este tratamiento puede evitar la recurrencia de cálculos salivales, aunque en otros se menciona que la existencia de una sialolitectomía no depende de realizar una sialodocoplastía según Park et al (30).

Kopec et al. (19) apuntan que también se puede colocar un catéter o estent (del inglés stent) de pequeño calibre en el conducto salival para evitar su estenosis; aunque ésta es una práctica que está descrita principalmente para el tratamiento de cálculos en el conducto de Stensen de la glándula parótida.

La resección quirúrgica de toda la glándula (sialodenectomía) puede ser una alternativa en casos severos, cuando hay evidencia de infección recurrente de la glándula y fibrosis, cuando el sialolito se encuentra ubicado muy posteriormente y cuando hay evidencia de una masa considerable dentro de la glándula establecen Marwaha \& Nanda (22), teniendo el riesgo de que permanezcan restos calcáreos en el conducto advierten Marchal \& Dulguerov (25).

Existen otras técnicas menos invasivas que se deben de tener en cuenta para disminuir la morbilidad de una cirugía. 
Purohit (4) menciona la litotripsia por onda de choque extracorpórea como una técnica alternativa, que consiste en fragmentar los sialolitos por medio de ondas expansivas, para que espontáneamente salgan del conducto sin lesionar tejidos adyacentes, disminuyendo la morbilidad, Kopec et al. (19) mencionan que este tratamiento podría requerir varias sesiones.

La sialendoscopía, o endoscopía de la glándula salival, es un método relativamente nuevo que permite examinar el epitelio que cubre los conductos y permite introducir una cesta para atrapar el sialolito, o introducir un láser para fragmentarlo (litotripsia por onda de choque intracorpórea); evitando escisiones innecesarias describe Baptista et al. (23), cuya principal limitación es que sirve únicamente para cálculos pequeños $(<4 \mathrm{~mm})$ según Saluja (15).

Izurieta etal. (1) menciona que otra alternativa es succionar el cálculo a través de un aspirador quirúrgico colocado en la desembocadura del conducto. Es útil en casos de sialolitos pequeños de poco calibre.

Una técnica también mínimamente invasiva y un poco más nueva es el uso de laser de C02, el cual es un procedimiento simple y seguro. La precisión del corte, la capacidad de penetración en el tejido que se detiene al contacto con el cálculo protege la mucosa impidiendo el daño ductal circunferencial y ausencia de sangrado, generando una mínima respuesta inflamatoria, ausencia de fibrosis que evita la necesidad de colocar sondas intraductales y permite recanalización del conducto sin riesgo de estenosis, con escasa posibilidad de infección, siendo más confortable para el paciente.

Con cualquier técnica que sea la que se seleccione se debe tener mucho cuidado con estructuras anatómicas vecinas, como por ejemplo lesiones al nervio lingual e hipogloso (riesgo de
$7 \%$ y nervio facial (riesgo de $12.5-30 \%$ en sialadenetomías), y también existe el riesgo de estenosis del conducto por sialolitectomía intraoral según Prado et al. (5).

Saluja et al. (15) menciona que se han reportado casos de recurrencia de sialolitiasis en periodos de hasta 10 años. Una dieta rica en proteínas y líquidos, incluyendo alimentos y bebidas ácidas que favorezcan el flujo salival, es recomendable para prevenir su recurrencia.

\section{CONCLUSIÓN}

La presencia de sialolitos en la glándula submandibular es una de las patologías más comunes de las glándulas salivales, aunque la formación de fístulas extraorales es muy rara. Debido a que es una afección muy asociada al dolor, inflamación e infección, su manejo debe ser muy meticuloso. Para el diagnóstico, es común el uso de radiografías intraorales y extraorales como la radiografía oclusal inferior y la panorámica, pero en casos de cálculos radiolúcidos otros métodos diagnósticos adicionales se pueden utilizar. Su tratamiento se puede realizar de diferentes maneras, desde un manejo conservador por medio de masajes, estimulación salival y prescripción de antiinflamatorios y antibióticos; hasta la resección quirúrgica del sialolito por cualquier método descrito, o en casos severos hasta la resección completa de la glándula afectada.

Es importante que todo odontólogo conozca la sintomatología, el diagnóstico y el tratamiento de esta patología, para brindar al paciente un tratamiento oportuno, aliviar el dolor agudo asociado y evitar complicaciones posteriores por no realizar un tratamiento a tiempo. Además, diagnosticar los casos en los que se debe abordar el tratamiento de manera conjunta con un especialista, todo siempre para beneficio del paciente. 


\section{REFERENCIAS}

1. Izurieta J, Sanabria M, Bastida D. (2009). Trastorno de las glándulas mayores. Sialolitiasis. Foros de Patología de la Universidad Rey Juan Carlos. 2009 [Citado 11 Oct 2014]. Disponible en: http://biopat.cs.urjc.es/ conganat/files/2009-2010_G17.pdf

2. Jiménez N, Reyes J.O. Sialolitiasis de la glándula submandibular. Reporte de caso. Med Oral 2000; 2: 76-79.

3. Escudier MP, McGurk M. Symptomatic sialoadenitis and sialolithiasis in the English population, an estimate of the cost of hospital treatment. British Dental Journal 1999; 186 (9): 463-466.

4. Purohit N, Raghavan V, Bedi S, Arora R. Recurrent Sialolithiasis-Report of an interesting case. Clinical Dentistry 2012; 6(5): 10-13.

5. Prado N, Prado J, Gonzalez, V, Gatica M. Sialolitectomía con laser de CO2. Reporte de Caso. Revista ADM 2011; 68(5): 249-253.

6. Okada H, Yokokawa MJ, Komiya M, Akimoto Y, Kaneda T, Yagamoto H. A rare case of sialolithiasis of the lower lip simulating a mucocele and review of the literature. Quintessence 2001; 42(7): 589-594.

7. Rebolledo M, Carbonell Z, Diaz A. Sialolitos en conductos y glándulas salivales. Revisión de literatura. Avances en Odontoestomatología 2009; 25(6): 311-317.

8. Iqbal A, Gupta AK, Natu SS, Gupta AK. Unusually large sialolith of Warthon's dut. Annals of Maxillofacial Surgery 2012; 2 (1):70-73.
9. Siddiqui SJ. Sialolithiasis: an unusually large submandibular salivary Stone. British Dental Journal 2002; 193(2):89-91.

10. Chandak R,DegwekarS,ChandakM, Rawlani S. Acute Submandibular Sialadenitis: A Case Report. Case Reports in Dentistry [Internet]. 2012. [citado 20 Sept 2014]. Disponible en http://dx.doi.org/10.1155/2012/615375.

11. Oteri G, Procopio R, Cicciu M. Giant Salivary Gland Calculi (GSGC). Report of two cases. The Open Dentistry Journal [Internet]. 2011 [citado 20 Sep 2014]; 5:90-95. Disponible en: http://benthamopen.com/TODENTJ/ VOLUME/5/.

12. Bernardon JL, Machado R, Lanes R, Lecher HO, Mirando R. Sialolito en glándula submandibular - relato de caso. Revista Faculdade de Odontologia da Universidade de Passo Fondo 2007; 12 (3): 61-64.

13. Teymoortash A, Tiemann M, Schrader C, Werner J. Characterization of lymphoid infiltrates in chronic obstructive sialadenitis associated with sialolithiasis. Journal Oral Pathological Medicine 2004; 33:300-304.

14. Prabhat MPV. Parothid Sialodenitis - Review and report of case. Annals of Essences of Dentistry 2011; 3(1): 65-69.

15. Saluja H, Kasat VO, Mahindra U. Giant Sialolith in Warthon's Duct causing sialooral fistula. J Orofacial Science 2012; 4(2):137-142.

16. Tomomichi O, Nomura J, Matsumura Y, Yanase S, Nagata T, Uno S, Tagawa T. A case of sialolithiasis in a child. The journal of pediatric dentistry 2006; 31(2): 139-141.

17. Crippa R, Calcagnile F. The use of laser technology for submandibular calculosis: a 
case report. Journal Oral Laser Applications 2003; 3(3):173-176.

18. Shetty BN, Sharma P. Unusual case of a projecting intraoral giant sialolith. Indian Journal of Surgery 2010; 72(2): 155-157.

19. Kopec T, Szyfter W, Wierzbicka M. Sialoendoscopy and combined approach for the management of salivary gland stones. European Archives of Oto-RhinoLaryngology 2013; 270: 219-223.

20. Peterson JR, Murphy MJ. Bones, groans, moan and salivary stones? Journal of Clinical Pathology 2001; 54(5):412.

21. Marchal F, Kurt AM, Dulguerov P, Lehmann W. Retrograde Theory in sialolithiasis formation. Archives of Otolaryngology- Head and Neck Surgery 2001; 127(1): 66-68.

22. Marwaha M, \& Nanda KS. Sialolithiasis in a 10 year old child. Indian Journal Dental Research 2012; 23(4):546-549.

23. Baptista P, Gimeno C, Rey JA, Casale M. Sialoendoscopy: A New Alternative for the Treatment of Salivary Pathology: Our Experience. Acta Otorrinolaringol Española 2008; 59(3):120-123.

24. Shingal A, Shingal P, Ram R, Gupta R. Self Exfoliation of large submandibular Stone- report of two cases. Contemporary Clinical Dentistry 2012; 3(2):185-187.

25. Marchal F, Dulguerov P. Sialolithiasis Management. The State of the Art. Arch Otolaryngol- Head Neck Surgery 2003; 129:951-956.

26. Williams MF. Sialolithiasis. Otolaryngologic Clinics of North America 1999; 32 (5): 819-834.

27. DalkizM, Dogan N,BeydemirB. Sialolithiasis (Salivary Stone). Turkish Journal of Medical Sciences 2001; 31:177-179.

28. Lemos-Junior C, Camargo A. Images in clinical medicine: Sialolith of the submandibular salivary gland. The New England Journal of Medicine 2011; 364 (6):551.

29. SreetharamSS, PhilipR.UnusualForeignBody of Parotid Gland Presenting as Sialolithiasis: Case Report and Literature Review. Case Reports in Otolaryngology [Internet]. 2012 [citado 06 Sep 2014]. Disponible en: http:// dx.doi.org/10.1155/2012/367349.

30. Park JH, Kim JW, Lee YM, Oh CW, Chang HS, LeeSW.Long-term Study of Sialodochoplasty for Preventing Submandibular Sialolithiasis Recurrence. Clinical and Experimental Otorhinolaryngology 2012; 5(1):34-38. 